\title{
Loss of ONECUT1 expression in human pancreatic cancer cells
}

\author{
XIAOHUA JIANG ${ }^{1,2 *}$, WEIWEI ZHANG ${ }^{1 *}$, HANY KAYED $^{3}$, PAN ZHENG $^{1}$, \\ NATHALIA A. GIESE ${ }^{3}$, HELMUT FRIESS ${ }^{1}$ and JÖRG KLEEFF ${ }^{1}$ \\ ${ }^{1}$ Department of Surgery, Technische Universität München, 81675 Munich, Germany; \\ ${ }^{2}$ Department of General Surgery, Zhongda Hospital, Southeast University, Nanjing 210009, P.R. China \\ ${ }^{3}$ Department of General Surgery, University of Heidelberg, 69120 Heidelberg, Germany
}

Received July 31, 2007; Accepted September 27, 2007

\begin{abstract}
ONECUT1 (HNF-6) is the prototype of a new class of homeodomain transcription factors, that controls the development of pancreatic ducts during mouse development. In the present study, the role of ONECUT1 and its targeted genes TCF2, PKHD1 and CYS1 was analyzed in human pancreatic ductal adenocarcinoma (PDAC). mRNA levels of ONECUT1, TCF2, PKHD1 and CYS1 were measured in pancreatic tissues and pancreatic cancer cell lines by quantitative reverse-transcriptase polymerase chain reaction (QRTPCR). Protein expression of ONECUT1 and TCF2 was assessed in pancreatic tissues by immunohistochemistry. ONECUT1 was transfected into Panc-1 and T3M4 pancreatic cancer cells and its effects on anchorage-dependent and independent growth as well as invasion and adhesion were analyzed. Median mRNA levels of ONECUT1, TCF2, PKHD1 and CYS1 were 7.7-, 2.0-, 5.7- and 3.8-fold higher in normal tissues than in PDAC tissues. ONECUT1 protein was expressed in normal acinar and ductal cells, but neither in the cancer cells of PDAC tissues nor in 7 of 8 cultured pancreatic cancer cell lines. There was a significant positive correlation between ONECUT1 and TCF2, CYS1, and PKHD1 mRNA levels in PDAC tissues. Transfection of ONECUT1 into pancreatic cancer cells resulted in up-regulation of the target gene TCF2, a reduction in invasiveness, but no change in adhesion or growth. In conclusion, ONECUT1 expression is lost in pancreatic cancer cells, suggesting a tumor suppressor function in this malignancy.
\end{abstract}

\section{Introduction}

Cilia are microtubule-based organelles projecting from the surface of cells, and are conserved in mammalian cells $(1,2)$. Cilia are classified into three basic categories: motile, primary

Correspondence to: Dr Jörg Kleeff, Department of Surgery, Technische Universität München, Ismaninger Strasse 22, 81675 Munich, Germany

E-mail:kleeff@t-online.de

\section{${ }^{*}$ Contributed equally}

Key words: ONECUT1, TCF2, CYS1, PKHD1, pancreatic cancer, invasion, cilia or nodal $(3,4)$. In the pancreas, cilia are observed in ductal and islet cells and possess the distinct $9+0$ microtubule pattern characteristic of primary cilia, which are thought to serve as mechanical and chemical sensors in the pancreas (3-6). It has been suggested that defects in the assembly and/or function of primary cilia cause cysts to appear. Insight into the molecular etiology of cystic diseases has been obtained mainly from studies of hereditary forms of polycystic kidney disease, and most mutations associated with cystic kidneys in humans or in animal models affect genes that code for proteins localized to the primary cilia $(7,8)$. Interestingly, pancreatic cysts are found in $10 \%$ of patients with polycystic kidney disease $(3,9)$. Recently, it has been shown that the transcriptional regulator ONECUT1 (hepatocyte nuclear factor 6, HNF-6) controls the development of inter- and intralobular pancreatic ducts in mice. Homozygous knockout of ONECUT1 in mice results in severe defects in pancreatic tubulogenesis during fetal life, leading to ducts with dramatically enlarged lumina and multiple cysts. A hallmark of cystic ducts in this novel model is the lack of primary cilia. Strikingly, mRNA levels of TCF2 (also known as HNF-13), fibrocystin/polyductin (encoded by the PKHD1 gene) and cystin (encoded by the CYS1 gene) are dramatically reduced in the pancreases of ONECUT1 knockouts (10). This shows that ONECUT1 controls a network of genes involved in primary cilia function $(10,11)$. Thus far, it is not known whether primary cilia play a role in carcinogenesis. Dysfunction of primary cilia can cause cystic disease $(12,13)$, and there seems to be a relationship between cilia, cell size and cell cycle progression (14). Interestingly, patients with autosomal dominant polycystic kidney disease can develop intraductal papillary mucinous neoplasm (IPMN) of the pancreas (15), which has the potential to progress to invasive pancreatic adenocarcinoma. To better elucidate the role of primary cilia in pancreatic carcinogenesis, in the present study we investigated the expression of ONECUT1 and its target genes TCF2, PKHD1 and CYS1 in the normal pancreas and pancreatic ductal adenocarcinoma.

\section{Materials and methods}

Tissue specimens. Pancreatic ductal adenocarcinoma tissue specimens $(n=79)$ were obtained from patients in whom pancreatic resections were carried out. Normal human pancreatic tissue samples $(n=26)$ were obtained through an 
organ donor program from previously healthy individuals. All samples were confirmed histologically. Freshly removed tissues were fixed in paraformaldehyde solution for 12-24 h and then paraffin embedded for histological analysis. In addition, a portion of the tissue samples was preserved in RNAlater (Ambion Europe Ltd., Huntingdon, Cambridgeshire, UK), or snap-frozen in liquid nitrogen immediately upon surgical removal and maintained at $-80^{\circ} \mathrm{C}$ until use. The Human Subjects Committee of the University of Heidelberg, Germany, approved the use of human tissues for molecular research. Written informed consent was obtained from all patients.

Cell culture. Pancreatic cancer cell lines were grown routinely in RPMI medium, supplemented with $10 \%$ fetal bovine serum, $100 \mathrm{U} / \mathrm{ml}$ penicillin, and $100 \mathrm{mg} / \mathrm{ml}$ streptomycin (Invitrogen, Karlsruhe, Germany). Cells were maintained at $37^{\circ} \mathrm{C}$ in a humidified atmosphere with $5 \% \mathrm{CO}_{2}$.

Real-time quantitative polymerase chain reaction. All reagents and equipment for mRNA/cDNA preparation were supplied by Roche Applied Science (Mannheim, Germany). Real-time quantitative PCR (QRT-PCR) was carried out using the LightCycler FastStart DNA SYBR Green kit, as described previously $(16,17)$. The number of specific transcripts was normalized to housekeeping genes [cyclophilin B (CPB) and hypoxanthine guanine phosphoribosyltransferase] and presented as adjusted transcripts per 10,000 CPB copies.

Laser capture microdissection (LCM) and microarray analysis. Human ductal cells were microdissected from normal pancreas $(n=3)$ and chronic pancreatitis $(n=5)$ samples as well as from pancreatic cancer cells of PDAC samples $(n=6)$. LCM and RNA extraction were carried out as described previously (18). The HG-U95Av2 array from Affymetrix (Santa Clara, CA) was used. Poly(A)+RNA isolation, cDNA synthesis, and cRNA in vitro transcription were performed as reported previously (19). Hybridization of the fragmented in vitro transcription products to oligonucleotide arrays was performed as suggested by the manufacturer (Affymetrix).

Immunohistochemistry. Paraffin-embedded tissue sections (3- $\mu \mathrm{m}$ thick) were deparaffinized in roticlear and rehydrated using routine methods $(16,17)$. After blocking of non-specific reactivity with normal goat serum (Kirkegaard \& Perry Laboratories, Inc., Gaithersburg, MD), sections were incubated with rabbit anti-human ONECUT1 (1:250) (Santa Cruz Bioscience, Santa Cruz, CA) or rabbit anti-human TCF2 (1:200) (Santa Cruz Bioscience) at $4^{\circ} \mathrm{C}$ overnight, or with normal rabbit $\operatorname{IgG}$ as a negative control. The slides were then incubated with biotinylated goat anti-rabbit antibody (Kirkegaard \& Perry Laboratories, Inc.) for $45 \mathrm{~min}$ at room temperature. Tissue sections were then incubated with streptavidin peroxidase (Kirkegaard \& Perry Laboratories, Inc.) for $45 \mathrm{~min}$ at room temperature. Each section was subjected to $100 \mu \mathrm{l}$ DAB-chromogen substrate mixture (Dako Corp., Carpinteria, CA) and then counterstained with Mayer's hematoxylin. Sections were washed, dehydrated in progressively increasing concentrations of ethanol, and mounted with xylene-based mounting medium. Slides were visualized using the Axioplan 2 imaging microscope (Carl Zeiss Lichtmicroskopie, Göttingen, Germany).

Transient transfection. Pancreatic cancer cells were seeded in 6-well plates and grown until 70-90\% confluence. For transient transfection, $4 \mu \mathrm{g}$ of the full-length ONECUT1 expression vector pCMV6-XL4-ONECUT1 (Origene, Rockville, MD) or $4 \mu \mathrm{g}$ of the control vector pCMV6-XL4 (Origene) were incubated with $10 \mu \mathrm{l}$ of lipofectamine 2000 in $500 \mu 1$ of RPMI medium for $20 \mathrm{~min}$ and added to the cells. After a 4-h incubation period, the medium was discarded and equal volumes of medium containing $10 \%$ fetal bovine serum was added and allowed to incubate for $12 \mathrm{~h}$. After $36 \mathrm{~h}$ of subsequent incubation in complete medium, cells were subjected to immunoblotting and functional assays.

Immunoblotting. Cells were washed twice with ice-cold PBS and lysed with lysis buffer (50 mM Tris- $\mathrm{HCl}, 100 \mathrm{mM} \mathrm{NaCl}$, $2 \mathrm{mM}$ EDTA, 1\% SDS) containing one tablet of complete mini-EDTA-free protease inhibitor cocktail (Roche Applied Science). Protein/lane $(20 \mu \mathrm{g})$ were separated on $4-12 \%$ SDS-polyacrylamide gels and transferred to PVDF membrane. Membranes were then incubated in $10 \mathrm{ml}$ blocking solution (5\% non-fat milk in $20 \mathrm{mM}$ Tris- $\mathrm{HCl}, 150 \mathrm{mM} \mathrm{NaCl}, 0.1 \%$ Tween-20), followed by incubation with the indicated antibody at $4^{\circ} \mathrm{C}$ overnight. Membranes were washed and incubated with horseradish peroxidase (HRP)-conjugated anti-rabbit antibody (Amersham International) for $1 \mathrm{~h}$ at room temperature. Equal loading was confirmed using a rabbit polyclonal ERK2 antibody (Santa Cruz Bioscience). Signals were detected using the enhanced chemiluminescence system (ECL, Amersham Life Science Ltd., Bucks, UK).

Adhesion assays. Pancreatic cancer cells were seeded in 96well plates $\left(2 \times 10^{4}\right.$ cells/well $)$ for the indicated time. After being washed 3 times with PBS, cells were stained with $100 \mu 1$ staining solution $(0.05 \%$ crystal violet in $20 \%$ ethanol) for $15 \mathrm{~min}$. Cells were washed, dried, and $50 \mu 1$ of $2 \%$ SDS was added per well. The number of adherent cells was determined by reading OD values at $570 \mathrm{~nm}$. All assays were performed in triplicate.

Invasion assays. BioCoat Matrigel (BD Biosciences, San Jose, CA) invasion chambers were rehydrated and reconstituted according to the manufacturer's instructions. Cells were trypsinized and seeded in the top chamber at a density of $5 \times 10^{4}$ cells/well in $600 \mu 1 \mathrm{RPMI}$ medium. After incubation for $24 \mathrm{~h}$, non-invading cells were removed from the upper surface of the separating membrane by gentle scrubbing with a cotton swab. Invading cells were fixed in $100 \%$ methanol and stained with $0.05 \%$ crystal violet in $20 \%$ ethanol. Membranes were mounted on glass slides and counted manually under a light microscope. The invasion index was calculated as the percentage of invaded cells in the treatment group compared to the percentage of invaded cells in the control group. All assays were performed in triplicate.

Statistical analysis. For statistical analyses, the non-parametric Mann-Whitney test was used, unless indicated otherwise. Significance was defined as $\mathrm{p}<0.05$. 

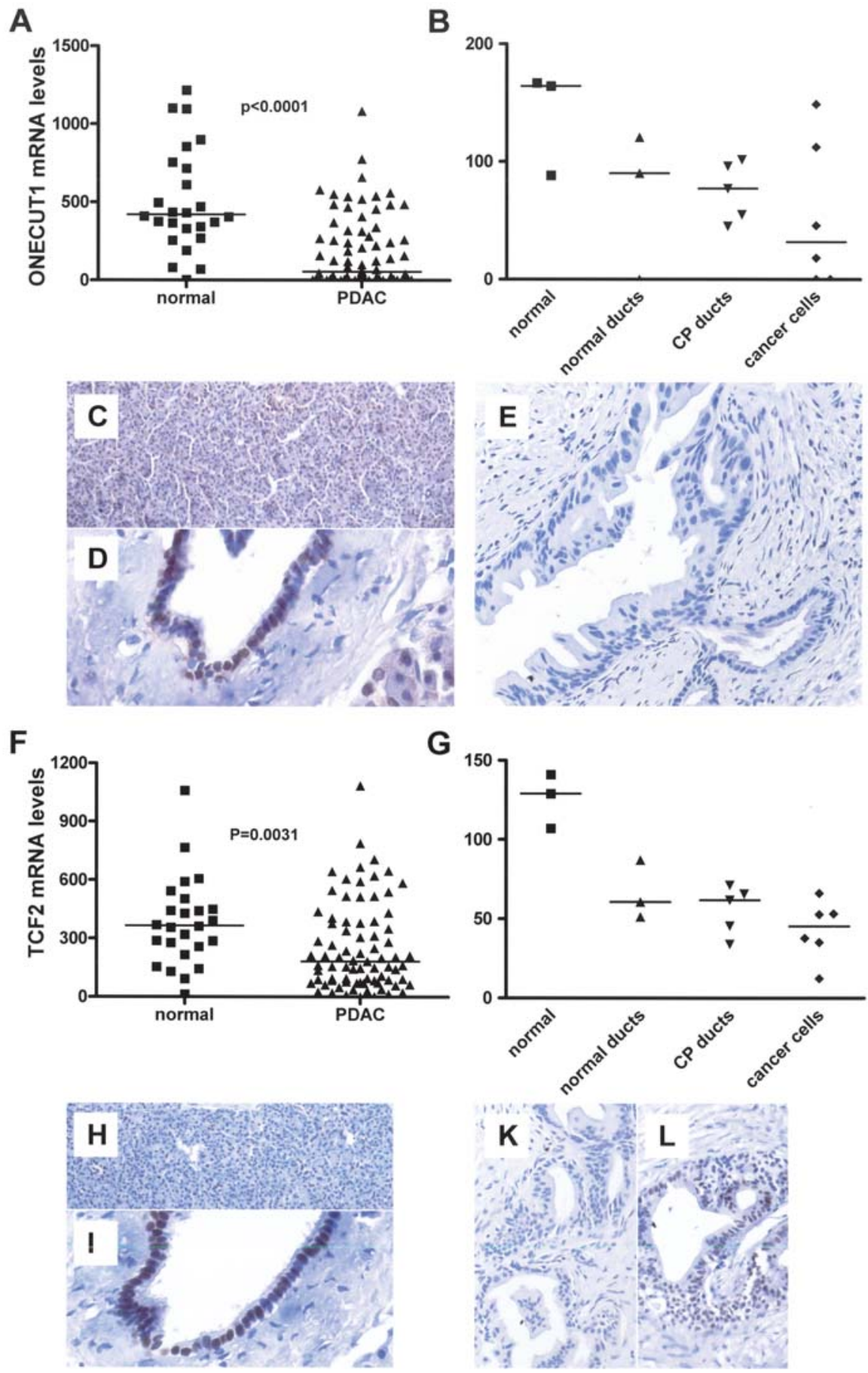

Figure 1. Expression of ONECUT1 and TCF2 in pancreatic tissues. mRNA expression levels of ONECUT1 and TCF2 in pancreatic tissues: real-time quantitative PCR analysis of mRNA levels for ONECUT1 (A) and TCF2 (F) in normal ( $\mathbf{\bullet}$ ) and PDAC ( $\mathbf{\Delta}$ ) tissue samples was performed as described in Materials and methods. Horizontal lines represent the median mRNA levels. RNA input was normalized to the average expression of the two housekeeping genes HPRT and CPB, and presented as adjusted transcripts/10,000 CPB copies. Microarray analysis of ONECUT1 (B) and TCF2 (G) in microdissected total normal pancreatic tissues $(\mathrm{n}=3)$, normal pancreatic ducts $(\mathrm{n}=3)$, ducts from chronic pancreatitis tissues $(\mathrm{n}=5)$, and cancer cells $(\mathrm{n}=6)$. Horizontal lines represent the median expression levels. Values are presented as arbitrary units. Localization of ONECUT1 and TCF2 in human pancreatic tissues: immunohistochemistry was performed as described in Materials and methods. Normal pancreatic tissues showed ONECUT1 staining in acini (C) and ducts (D) and TCF2 staining in acini $(\mathrm{H})$ and ducts (I). ONECUT1 staining was absent (E) in cancer cells of PDAC tissues, while TCF2 staining was absent (K) or moderate (L).

\section{Results}

ONECUT1 expression and localization in human pancreatic tissues. Quantitative RT-PCR was performed to evaluate the mRNA levels of ONECUT1 expression in normal pancreatic tissue samples $(n=26)$ and PDAC samples $(n=79)$. Tissue samples from normal pancreases had a median (range) replicate number of ONECUT1 mRNA of 421 (3-1846), in contrast to $55(0-1556)$ in PDAC $(\mathrm{p}<0.0001)$ (Fig. 1A). To determine whether the low expression of ONECUT1 in PDAC tissues was specifically due to loss of expression in cancer cells, a microarray analysis of microdissected pancreatic ductal cells from the normal pancreas and cancer cells as well as of bulk normal pancreatic tissues was performed. This analysis revealed that 4 of 6 cases of microdissected cancer cells expressed ONECUT1 below the range of both microdissected 
A

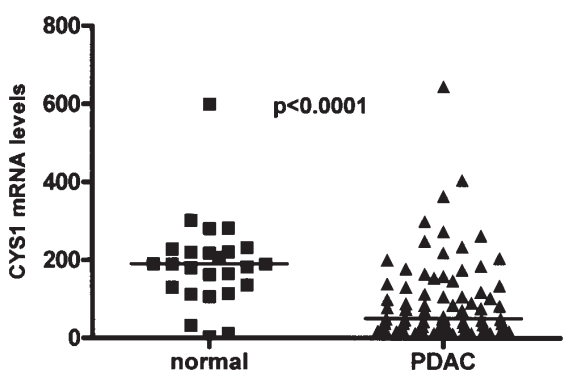

B

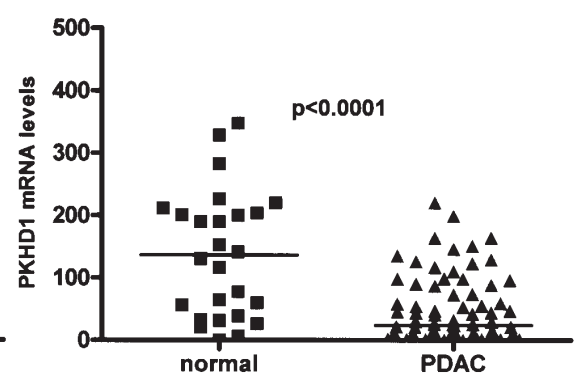

C

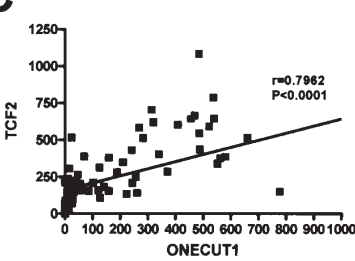

D

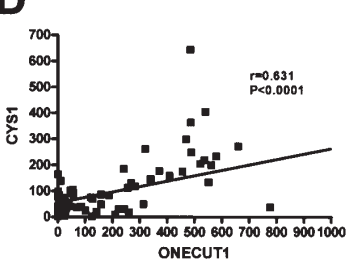

E

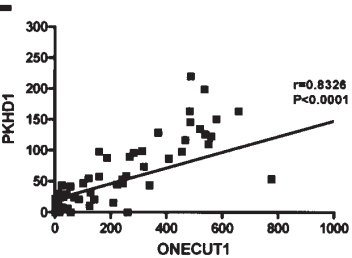

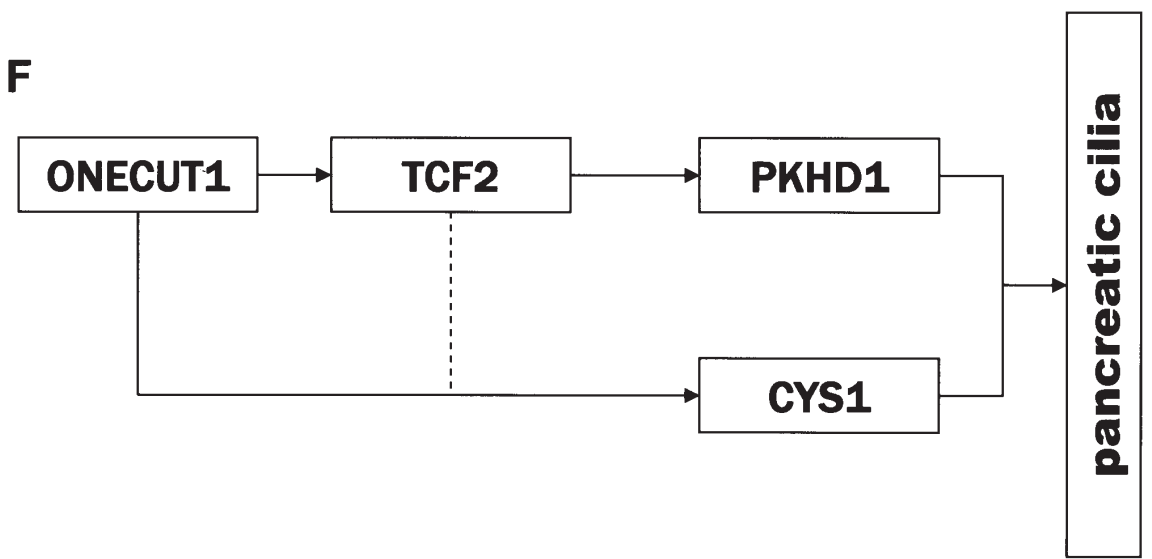

Figure 2. Expression of CYS1 and PKHD1 in pancreatic tissues. mRNA expression levels of CYS1 and PKHD1 in pancreatic tissues: real-time quantitative PCR

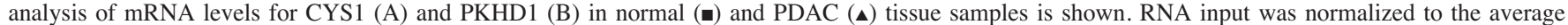
expression of the two housekeeping genes HPRT and CPB, and is presented as adjusted transcripts/10,000 CPB copies. Correlation of ONECUT1 mRNA expression levels with TCF2, CYS1 and PKHD1: correlation analysis showing significant correlation of ONECUT1 mRNA levels with TCF2 (C), CYS1 (D) and PKHD1 (E) is shown. Proposed transcription factor network controlling differentiation of pancreatic ducts: ONECUT1 is required for the activation of genes that establish normal epithelial polarity. In addition, ONECUT1 also regulates components of the primary cilium, such as CYS1 and PKHD1. While CYS1 is likely controlled by ONECUT1 directly, the activation of PKHD1 involves a transcription factor cascade with TCF2 (F). Adapted from Kaestner et al (11).

normal pancreatic ducts and bulk normal pancreatic tissues (Fig. 1B). Immunohistochemistry was performed next to determine the distribution of ONECUT1 expression in normal pancreatic and PDAC tissues. Ductal and acinar cells in all 10 normal pancreatic tissues exhibited ONECUT1 immunostaining (Fig. 1C and D). In contrast, ONECUT1 expression was not present in cancer cells in any of the 27 PDAC tissues (Fig. 1E).

TCF2 expression and localization in human pancreatic tissues. Next, the expression of ONECUT1 downstream targets was analyzed. Tissue samples from normal pancreases had a median (range) replicate number of TCF2 mRNA of 365.5 (13-1692), while it was $182.0(1-1084)$ in PDAC ( $\mathrm{p}=0.031)$ (Fig. 1F). Microarray analysis revealed that 5/6 microdissected cancer cells expressed TCF2 below the range of both microdissected normal pancreatic ducts and bulk normal pancreatic tissues (Fig. 1G). Immunohistochemistry demonstrated that the ductal and acinar cells in all 10 normal pancreatic tissues exhibited TCF2 immunostaining (Fig. 1H and I). In contrast, only 15 of 29 PDAC tissues (52\%) exhibited TCF2 immunoreactivity in the cancer cells (Fig. $1 \mathrm{~K}$ and $\mathrm{L}$ ).

CYS1 and PKHD1 mRNA expression in human pancreatic tissues. When we further investigated the target genes CYS1 and PKHD1, the same tendency - i.e., down-regulation in cancer - was observed. PDAC tissue samples had a median (range) replicate number of CYS1 mRNA of 50.0 (3-645) and PKHD1 mRNA of 24.0 (0-220) compared with 190.0 (4-938) and 136.5 (0-348), respectively, in the normal pancreas $(\mathrm{p}<0.0001)$ (Fig. 2A and B).

Correlation of ONECUT1 with TCF2, CYS1 and PKHD mRNA levels in PDAC tissues. As shown above, there was down- 
A

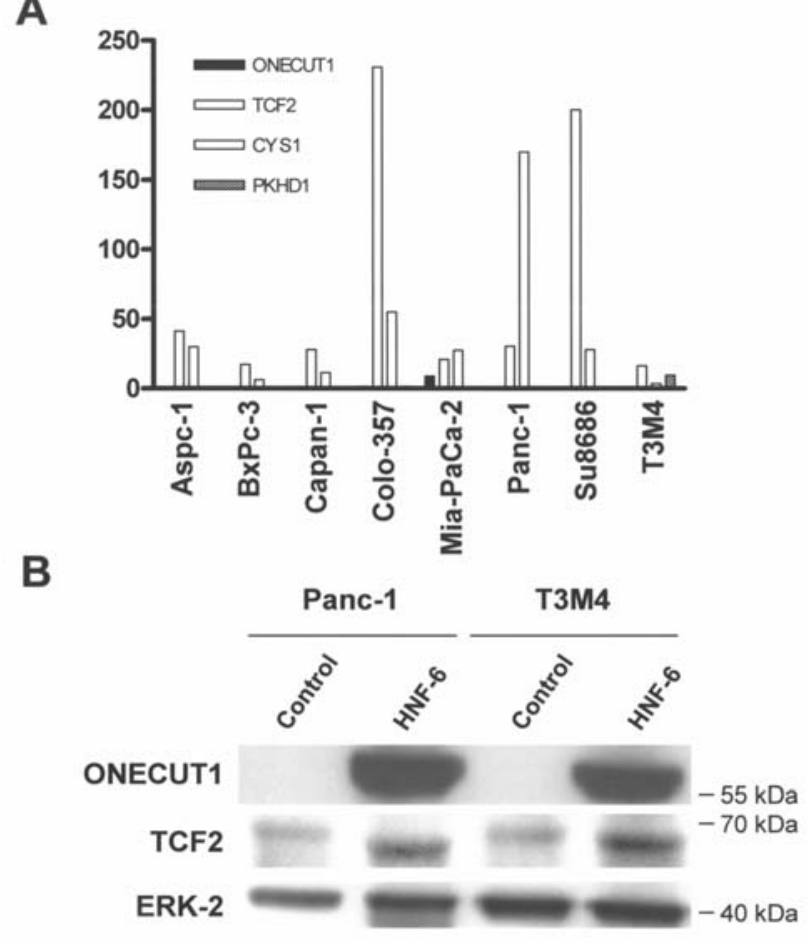

C

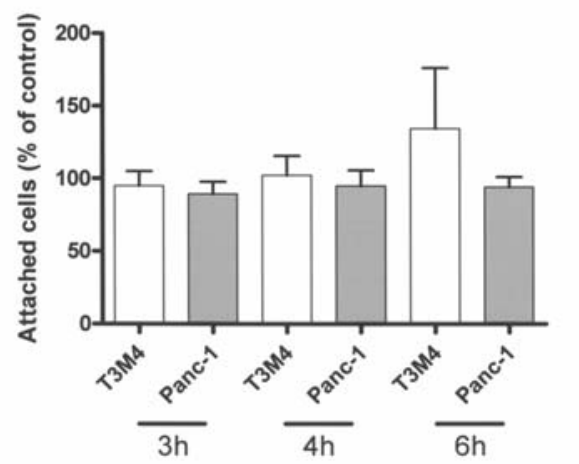

D

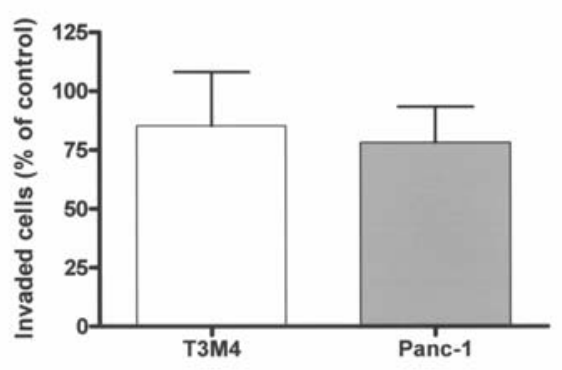

Figure 3. Expression and function of ONECUT1, TCF2, CYS1 and PKHD1 in pancreatic cancer cell lines. Expression of ONECUT1, TCF2, CYS1 and PKHD1 mRNA levels in pancreatic cancer cell lines (A): real-time quantitative PCR analysis of ONECUT1, TCF2, CYS1 and PKHD1 mRNA levels in pancreatic cancer cell lines was performed as described in Materials and methods. RNA input was normalized to the average expression of the two housekeeping genes HPRT and CPB, and presented as adjusted transcripts/10,000 CPB copies. ONECUT1 overexpression and its effects on TCF2 in human pancreatic cancer cells (B): Panc-1 and T3M4 pancreatic cancer cell lines were transfected with a ONECUT1 expression vector or control vector for 48 h, and cell lysates were subjected to immunoblotting, as described in Materials and methods. ONECUT1 expression (upper panel) and TCF2 expression (middle panel) were analyzed by immunoblotting. Equal loading was determined using an ERK-2 antibody (lower panel). Impact of ONECUT1 overexpression on adhesion and invasion of pancreatic cancer cells: Panc-1 and T3M4 pancreatic cancer cell lines were transfected with a ONECUT1 expression vector or a control vector for 48 h. Adhesion of cancer cells after ONECUT1 transfection was recorded as a percentage of control. Results at different time-points $(3,4$ and $6 \mathrm{~h})$ are presented as mean \pm SEM of three independent experiments (C). Invasiveness of cancer cells after ONECUT1 transfection was recorded as a percentage of control. Results are presented as mean \pm SEM of three independent experiments (D).

regulation of ONECUT1, TCF2, CYS1 and PKHD mRNA levels in PDAC. Since TCF2, CYS1 and PKHD are all targets of ONECUT1, we next analyzed whether there is a correlation between ONECUT1 and TCF2, CYS1 and PKHD mRNA levels in PDAC tissues. There was significant positive correlation of ONECUT1 with TCF2 $(r=0.796, \mathrm{p}<0.0001)$, CYS1 $(\mathrm{r}=0.631, \mathrm{p}<0.0001)$, and PKHD1 $(\mathrm{r}=0.833, \mathrm{p}<0.0001)$ (Fig. 2C-E). There was also significant correlation of TCF2 with CYS $1(\mathrm{r}=0.653, \mathrm{p}<0.0001)$ and PKHD1 ( $\mathrm{r}=0.801$, $\mathrm{p}<0.0001$ ) (data not shown). These results provide indirect evidence that ONECUT1 also regulates components of the primary cilium, such as CYS1 and PKHD1 (Fig. 2F).

ONECUT1, TCF2, CYS1 and PKHD1 mRNA expression in pancreatic cancer cell lines. mRNA levels of ONECUT1, TCF2, CYS1 and PKHD1 were analyzed by QRT-PCR in 8 pancreatic cancer cell lines (Fig. 3A). Both ONECUT1 and PKHD1 mRNA expression levels were very low in all 8 pancreatic cancer cell lines (0-9 and 0-9.7 copies/10,000 CPB respectively). CYS1 expression varied in different cell lines, 
from 3 copies/10,000 CPB in T3M4 to 170 copies/10,000 CBP in Panc-1 cells. TCF2 expression was the highest among the 4 genes. Interestingly, relatively high copy numbers were observed in the cell lines Colo-357 (231 copies/10,000 CPB) and Su8686 (200 copies/10,000 CPB), which did not express ONECUT1 mRNA.

Effects of ONECUT1 overexpression on adhesion and invasion in pancreatic cancer cells. Since ONECUT1 is expressed only in normal pancreatic ducts but not in pancreatic cancer cells, we hypothesized that during pancreatic carcinogenesis, ONECUT1 expression is lost. To further analyze the effects of ONECUT1 in pancreatic cancer cells, we overexpressed ONECUT1 in T3M4 and Panc-1 cells by transfection with an ONECUT1 expression vector. Immunoblotting confirmed successful transfection and adequate expression of ONECUT1 in both cell lines (Fig. 3B). The downstream target TCF2 was also induced in ONECUT1-transfected pancreatic cancer cell lines (Fig. 3B). Adhesion assays showed no significant difference between ONECUT1-transfected cells and cells transfected with the control vector (Fig. 3C). In contrast, invasion assays revealed a slight but significant decrease of invasiveness after ONECUT1 re-expression in Panc-1 (-21.7 $14.8 \%)$ $(\mathrm{p}<0.05)$ but not in T3M4 cancer cells $(-14.7 \pm 22.9 \%)$ (Fig. 3D).

\section{Discussion}

ONECUT1 (HNF-6) belongs to the new ONECUT class of cut homeodomain proteins, whose members contain a single cut domain and a divergent homeodomain $(20,21)$. In the mouse embryo, ONECUT1 is expressed in several tissues, including the epithelial cells of the pancreas, starting at the onset of its development. During formation of the acini, ducts, and islets, the expression of ONECUT1 becomes restricted to the acini and ductal cells $(22,23)$. Thus, the sequential activation of ONECUT1 in the endoderm controls the generation of pancreatic precursors (24). In the absence of ONECUT1, cysts appear within the interlobular and intralobular ducts. This is associated with abnormal development of primary cilia at the apical pole of the ductal cells and with reduced expression of a set of genes involved in polycystic diseases, namely those encoding for TCF2 and for the ciliumassociated proteins polyductin/fibrocystin and cystin. Primary cilia deletion in pancreatic epithelial cells results in ductal metaplasia, which appears to be due to expansion of ductal cells rather than to transdifferentiation of acinar cells (6).

In the present study, we investigated the role of the ONECUT transcription factor ONECUT1 in pancreatic ductal adenocarcinoma (PDAC). We showed that ONECUT1 mRNA expression is decreased in PDAC tissues compared to normal pancreatic tissues. Microarray analysis showed lower ONECUT1 levels in pancreatic cancer cells than in the normal pancreas and pancreatic ductal cells. Immunohistochemistry demonstrated that ONECUT1 was localized in acinar cells and normal pancreatic ductal cells. Absent ONECUT1 staining in pancreatic cancer cells matched the low ONECUT1 mRNA levels. In addition, very low to absent ONECUT1 mRNA levels in all cultured pancreatic cancer cell lines confirmed the loss of ONECUT1 expression in pancreatic cancer cells.

As a transcription factor, ONECUT1 controls a network of genes involved in primary cilia function, including TCF2,
CYS1 and PKHD1 (reviewed in ref. 11). This prompted us to examine the expression of genes targeted by ONECUT1. QRT-PCR showed that mRNA levels of TCF2 were significantly lower in PDAC tissues than in normal pancreatic tissues. Immunohistochemistry revealed that TCF2 was also expressed in acinar and ductal cells in normal pancreatic tissues. In contrast to ONECUT1, TCF2 was detected in the cancer cells of 15 of 29 PDAC tissues. In addition, QRT-PCR in pancreatic cancer cell lines demonstrated moderate TCF2 expression in Colo-357 and SU8686 cell lines even in the absence of ONECUT1 expression. These results indicate that loss of ONECUT1 expression does not result in loss of TCF2 expression in all cases. Thus, TCF2 seems to be regulated in part by other factors, as has been shown previously (25). CYS1 and PKHD1 mRNA levels were also significantly lower in PDAC tissues than in normal pancreatic tissues. To further prove that expression of TCF2, CYS1 and PKHD1 was at least partially regulated by ONECUT1, ONECUT1 levels were correlated with TCF2, CYS1 and PKHD1 mRNA levels. There were positive correlations between ONECUT1 and these three target genes.

In the normal pancreas, ONECUT1 participates in the formation of normal pancreatic ducts and controls the differentiation of ductal cells. We hypothesized that during the process of pancreatic cancer carcinogenesis, ONECUT1 expression is lost, and that this loss of expression contributes to disease aggressiveness.

ONECUT1 re-expression resulted in increased TCF2 levels, confirming that this gene is regulated in part by ONECUT1. ONECUT1 expression did not influence anchorage-dependent or anchorage-independent cell growth (data not shown), and did not affect cell adhesiveness. In contrast, ONECUT1 overexpression resulted in decreased invasiveness of pancreatic cancer cells. This is in agreement with similar effects that have been observed for another member of the HNF family (HNF4) in hepatocellular carcinoma (26). Thus, forced expression of HFN4 promotes reversion of tumors towards a less invasive and highly differentiated phenotype.

In conclusion, the present data show that ONECUT1 expression is lost in pancreatic cancer cells. This loss of expression, together with ONECUT1's effects on tumor invasion, suggests that ONECUT1 might act as a tumor suppressor in pancreatic cancer.

\section{References}

1. Bisgrove BW and Yost HJ: The roles of cilia in developmental disorders and disease. Development 133: 4131-4143, 2006.

2. Oishi I, Kawakami Y, Raya A, Callol-Massot C and Izpisua Belmonte JC: Regulation of primary cilia formation and leftright patterning in zebrafish by a non-canonical Wnt signaling mediator, duboraya. Nat Genet 38: 1316-1322, 2006.

3. Davenport JR and Yoder BK: An incredible decade for the primary cilium: a look at a once-forgotten organelle. Am J Physiol Renal Physiol 289: F1159-F1169, 2005.

4. Marshall WF and Nonaka S: Cilia: tuning in to the cell's antenna. Curr Biol 16: R604-R614, 2006.

5. Aughsteen AA: The ultrastructure of primary cilia in the endocrine and excretory duct cells of the pancreas of mice and rats. Eur J Morphol 39: 277-283, 2001.

6. Cano DA, Sekine S and Hebrok M: Primary cilia deletion in pancreatic epithelial cells results in cyst formation and pancreatitis. Gastroenterology 131: 1856-1869, 2006. 
7. Tahvanainen E, Tahvanainen $P$, Kaariainen $H$ and Hockerstedt K: Polycystic liver and kidney diseases. Ann Med 37: 546-555, 2005.

8. Torres VE and Harris PC: Mechanisms of disease: autosomal dominant and recessive polycystic kidney diseases. Nat Clin Pract Nephrol 2: 40-55, 2006.

9. Cano DA, Murcia NS, Pazour GJ and Hebrok M: Orpk mouse model of polycystic kidney disease reveals essential role of primary cilia in pancreatic tissue organization. Development 131: 3457-3467, 2004.

10. Pierreux CE, Poll AV, Kemp CR, Clotman F, Maestro MA, Cordi S, Ferrer J, Leyns L, Rousseau GG and Lemaigre FP: The transcription factor hepatocyte nuclear factor- 6 controls the development of pancreatic ducts in the mouse. Gastroenterology 130: 532-541, 2006.

11. Kaestner KH: Of cilia and cysts: modeling pancreatic polycystic disease. Gastroenterology 130: 926-928, 2006.

12. Christensen ST, Pedersen LB, Schneider L and Satir P: Sensory cilia and integration of signal transduction in human health and disease. Traffic 8: 97-109, 2007.

13. Ibraghimov-Beskrovnaya O: Targeting dysregulated cell cycle and apoptosis for polycystic kidney disease therapy. Cell Cycle 6: 776-779, 2007.

14. Quarmby LM and Parker JD: Cilia and the cell cycle? J Cell Biol 169: 707-710, 2005.

15. Naitoh H, Shoji H, Ishikawa I, Watanabe R, Furuta Y, Tomozawa S, Igarashi H, Shinozaki S, Katsura H, Onozato R and Kudoh M: Intraductal papillary mucinous tumor of the pancreas associated with autosomal dominant polycystic kidney disease. J Gastrointest Surg 9: 843-845, 2005.

16. Erkan M, Kleeff J, Esposito I, Giese T, Ketterer K, Buchler MW, Giese NA and Friess H: Loss of BNIP3 expression is a late event in pancreatic cancer contributing to chemoresistance and worsened prognosis. Oncogene 24: 4421-4432, 2005.

17. Kayed H, Kleeff J, Kolb A, Ketterer K, Keleg S, Felix K, Giese T, Penzel R, Zentgraf H, Buchler MW, Korc M and Friess H: FXYD3 is overexpressed in pancreatic ductal adenocarcinoma and influences pancreatic cancer cell growth. Int J Cancer 118: 43-54, 2006.

18. Ketterer K, Rao S, Friess H, Weiss J, Buchler MW and Korc M: Reverse transcription-PCR analysis of laser-captured cells points to potential paracrine and autocrine actions of neurotrophins in pancreatic cancer. Clin Cancer Res 9: 5127-5136, 2003.
19. Friess H, Ding J, Kleeff J, Fenkell L, Rosinski JA, Guweidhi A, Reidhaar-Olson JF, Korc M, Hammer J and Buchler MW: Microarray-based identification of differentially expressed growth- and metastasis-associated genes in pancreatic cancer. Cell Mol Life Sci 60: 1180-1199, 2003.

20. Lannoy VJ, Burglin TR, Rousseau GG and Lemaigre FP: Isoforms of hepatocyte nuclear factor-6 differ in DNA-binding properties, contain a bifunctional homeodomain, and define the new ONECUT class of homeodomain proteins. J Biol Chem 273: 13552-13562, 1998.

21. Lemaigre FP, Durviaux SM, Truong O, Lannoy VJ, Hsuan JJ and Rousseau GG: Hepatocyte nuclear factor 6, a transcription factor that contains a novel type of homeodomain and a single cut domain. Proc Natl Acad Sci USA 93: 9460-9464, 1996.

22. Landry C, Clotman F, Hioki T, Oda H, Picard JJ, Lemaigre FP and Rousseau GG: HNF-6 is expressed in endoderm derivatives and nervous system of the mouse embryo and participates to the cross-regulatory network of liver-enriched transcription factors. Dev Biol 192: 247-257, 1997.

23. Rausa F, Samadani U, Ye H, Lim L, Fletcher CF, Jenkins NA, Copeland NG and Costa RH: The cut-homeodomain transcriptional activator HNF-6 is coexpressed with its target gene HNF-3 beta in the developing murine liver and pancreas. Dev Biol 192: 228-246, 1997.

24. Poll AV, Pierreux CE, Lokmane L, Haumaitre C, Achouri Y, Jacquemin P, Rousseau GG, Cereghini S and Lemaigre FP: A vHNF1/TCF2-HNF6 cascade regulates the transcription factor network that controls generation of pancreatic precursor cells. Diabetes 55: 61-69, 2006.

25. Odom DT, Zizlsperger N, Gordon DB, Bell GW, Rinaldi NJ, Murray HL, Volkert TL, Schreiber J, Rolfe PA, Gifford DK, Fraenkel E, Bell GI and Young RA: Control of pancreas and liver gene expression by $\mathrm{HNF}$ transcription factors. Science 303: 1378-1381, 2004.

26. Lazarevich NL, Cheremnova OA, Varga EV, Ovchinnikov DA, Kudrjavtseva EI, Morozova OV, Fleishman DI, Engelhardt NV and Duncan SA: Progression of HCC in mice is associated with a down-regulation in the expression of hepatocyte nuclear factors. Hepatology 39: 1038-1047, 2004. 